\title{
Compression behavior and energy absorption of carbon fiber reinforced composite sandwich panels made of three -dimensional honeycomb grid cores
}

\author{
Jian Xiong ${ }^{\text {a* }}$, Ashkan Vaziri ${ }^{\mathrm{b}}$, Ranajay Ghosh ${ }^{\mathrm{b}}$, Hong $\mathrm{Hu}^{\mathrm{c}}{ }^{\mathrm{c}}$, Li Ma ${ }^{\mathrm{a}}$, and Linzhi Wu ${ }^{\mathrm{a}}$ \\ ${ }^{\text {a }}$ Center for Composite Materials and Structures, Harbin Institute of Technology, \\ Harbin 150001, China \\ ${ }^{\mathrm{b}}$ Department of Mechanical and Industrial Engineering \\ Northeastern University, Boston, MA 02115 USA \\ ${ }^{c}$ Institute of Textiles and Clothing, The Hong Kong Polytechnic University, China
}

\begin{abstract}
:
Carbon fiber reinforced three dimensional egg and pyramidal honeycomb grids cores with interconnected void spaces were fabricated using an interlocking method. The out-of- plane compression properties, failure modes, and energy absorption capacity of all-composite sandwich panels made of the new 3D grid cores were investigated. The analytical models for predicting the compressive stiffness and strength of both egg and pyramidal honeycomb grids cores were derived. The results showed that the fabricated sandwich panels have higher specific energy absorbing abilities compared to lightweight square honeycombs of same density $\left(10-100 \mathrm{Kg} / \mathrm{m}^{3}\right)$. The new core design promises novel applications for lightweight multifunctional structures due to increased flow in the inner spaces of the core construction, embedding of electrical lines, enhanced heat transfer, fuel storage and higher energy absorption compared to traditional cores. Keywords: Carbon fiber sandwich; Three dimensional grid; Interlocking; Multifunctional.
\end{abstract}

\section{Introduction}

Sandwich panels have found wide structural applications due to their light weight, high bending stiffness and high energy absorption ability. Traditional sandwich panels are made from foam and honeycomb cores with close-cells thereby impeding their deployment as functional structures [1-3]. In contrast, sandwich panels with open cell cores, in addition to high specific strength and high specific stiffness also exhibit multifunctional benefits, such as fluid circulation [4-6], lines embedding [7], active cooling [8], energy absorption characteristics [9] and

\footnotetext{
${ }^{*}$ Corresponding author, Tel.:+86 451 86412549; fax: +86 45186402386.

E-mail address: jx@hit.edu.cn (Jian Xiong)
} 
electromagnetic wave absorption [10] making them promising candidates for functional applications. To this end, using fiber reinforced composites as the core material can further improve the performance and design envelopes since they can potentially increase the specific strength and stiffness of sandwich structures. However, in spite of this promise, fabrication remains a key challenge due to the inherent geometrical and material complexity of these structures. To this end, an interlocking method has been employed to fabricate metal square honeycombs structures suitable for multiple functions such as energy absorption [11-12], sound transmission [13-14] and thermal management [15]. However, composites resist a straightforward extension of this technique. For instance, although the interlocked cores constructed from the carbon fiber reinforced Kagome and square grids were manufactured and tested [16-19], so far only two-dimensional (2D) grid cores have been fabricated through the interlocking method. In order to truly harness multifunctionality, fabrication of three-dimensional (3D) carbon fiber reinforced grid cores, which combine the advantages of both 2D grids and hollow cores with interconnected void spaces, is imperative.

This letter reports the manufacturing of 3D carbon fiber reinforced honeycomb cores of two types - egg honeycomb grid core and pyramidal honeycomb grid core, fabricated by an interlocking method. In addition, out-of-plane compression and energy absorption behavior of the sandwich panels were investigated. The particular geometry of the fabricated cores can lead to an increased flow in the inner spaces and higher energy absorption than traditional grid cores at low density. The proposed core constructions are promising candidates for a number of lightweight multifunctional applications.

\section{Experimental}

\subsection{Fabrication of 3D sandwich cores and panels}

In this work two types of 3D core constructions, namely egg honeycomb grid core and pyramidal honeycomb grid core were fabricated with carbon fiber reinforced composite sheets by using the plate interlocking method, Fig. 1 . The composites sheets used were $0^{\circ} / 90^{\circ}$ composite laminates made from $0.15 \mathrm{~mm}$ thickness T700/epoxy prepreg provided by Beijing Institute of Aeronautical Materials in China (The properties of the unidirectional laminate can be found in our previous paper [16]). They were first cut into two types of grid plates (Plate I and Plate II) as 
shown in Fig. 1(a) by using an electronic engraving machine built by Harbin Weijifen Organic Glass Products Co., Ltd. The cutting distance of the caulking grooves equals the thickness of the composite sheets, and two types of caulking grooves, i.e., grooves with long cuts and grooves with short cuts, were adopted. Then, the cut plates were assembled together through an interlocking method to form 3D sandwich cores. While the egg grid core was formed by interlocking two types of cut plates through the mechanical snap-fitting at the long grooves, the pyramidal grid core was formed by interlocking two types of cut plates through the mechanical snap-fitting at both the short and long grooves, Fig. 1(b). The unit-cells of both the egg honeycomb grid and pyramidal honeycomb grid cores are shown in Fig. 1(c). The photographs of two types of 3D grid cores fabricated are shown in Fig.1 (d).

As shown in Fig. 1(c), $a, b, H, h, t, d$ and $\omega$ are the geometrical parameters which determine the shape and size of cores. In this study, all the parameters were fixed except $d$ and were set to: $a=$ $45.51 \mathrm{~mm}, b=9.51 \mathrm{~mm}, H=20 \mathrm{~mm}, h=8 \mathrm{~mm}, \omega=45^{\circ}$ and $t=6 \mathrm{~mm}$. For $d$, two different values, i.e., $d=1 \mathrm{~mm}$ and $2 \mathrm{~mm}$, were chosen corresponding to two different thicknesses of carbon fiber reinforced composite sheets. By combining two thicknesses and two grid cores, in total four different cores were manufactured. The relative density of the egg honeycomb grid core, denoted by $\bar{\rho}$, can be approximated as,

$$
\bar{\rho}=\frac{d[(b+a-2 t)(H-h)+2 a h-d]}{a^{2} H}
$$

Due to the structural feature of the construction, the relative density of the pyramidal honeycomb grid core is double that of the egg honeycomb grid core if the same cut composite sheets are used. Based on the above geometrical parameters, the relative densities calculated for the egg grid cores are 0.03 and 0.06 and those of the pyramidal honeycomb grid cores are 0.06 and 0.12 , respectively.

The sandwich panels were assembled by bonding the carbon fiber reinforced composite face sheets to both the top and bottom sides of the fabricated grid cores by using an adhesive 08-57 provided by Heilongjiang Institute of Petrochemical. The assembled composite structure was finally heated and cured at a constant temperature of $120{ }^{\circ} \mathrm{C}$ for 1 hour thereby completing the fabrication. All the face sheets used were from the same $1.0 \mathrm{~mm}$ thickness fiber reinforced 
laminate sheets made with a stacking sequence $\left[0^{0} / 90^{0} / 90^{0} / 0^{0}\right]_{2}$. Fig. 2 shows a sandwich panel with the pyramidal honeycomb grid core made from $2 \mathrm{~mm}$ thickness carbon fiber reinforced composite sheets.

\subsection{Out-of-plane compression tests}

The out-of-plane compression tests on the fabricated specimens were carried out using a screw-driven testing machine (INSTRON 5569) according to ASTM C365/C 364M-05 at a nominal displacement rate of $0.5 \mathrm{~mm} / \mathrm{min}$. All the specimens were prepared with $3 \times 3$ unit cells. Therefore, the dimensions of the panel made of egg honeycomb grid cores were $136.5 \mathrm{~mm} \times$ $136.5 \mathrm{~mm}$, and those of the panel made of pyramidal honeycomb grid cores were $96.5 \mathrm{~mm} \times 96.5$ $\mathrm{mm}$. All the specimens were placed between two square steel plates with dimensions of $200 \mathrm{~mm}$ $\times 200 \mathrm{~mm} \times 20 \mathrm{~mm}$ (thickness) during static compression tests. The applied loads were measured via a load cell of the testing machine and the nominal axial strains of the specimens were measured with a laser extensometer.

\section{Results and discussion}

\subsection{Compression response}

The out-of-plane compression stress-strain curves of both egg and pyramidal honeycomb grid sandwich panels with different relative densities of cores are plotted in Fig. 3 (a) and 4 (a). In general, all the curves exhibited similar form with three distinct stages of deformation: initial linear compression, plateau and densification. In addition, two peak strengths were found on each curve. The first peak strength coincides with the failure of the top of the trapezium shaped grid core plates, Fig. 1, and was followed by plateau stage of response. Thereafter, the second peak strength occurred at the complete crushing failure of the remaining portion. In spite of these similarities, several distinctions were also clear. For instance, the sandwich panels made with the same type of cores exhibited higher compression strength for higher relative densities. On the

other hand, for the sandwich panels made with different cores, it was found that the compressive strength of pyramidal honeycomb grid sandwich panel was slightly higher than that of egg 
honeycomb grid sandwich panel for the same relative density $(\bar{\rho}=6.0 \%)$. This difference can be attributed to the fact that the pyramidal honeycomb grid core is formed with more interlocking points than egg honeycomb grid core for the same area, thereby improving its stability over the egg honeycomb grid core, which leads to an increase in strength of the sandwich panel with pyramidal honeycomb grid core.

The typical failure modes of the sandwich panels made of the same thickness of cut composite sheets $(d=1 \mathrm{~mm}$ and $2 \mathrm{~mm})$, but with different types of grid cores under the out-of-plane compression are shown in Figs. 3 and 4, respectively. Both delamination and fracture could be found near the top end of grid cores and these failures run through all the grid cores with increasing compressive loads. The delamination process of grid cores can be visualized as flowering shaped by the two halves of the laminate. This gradual flowering mode of failure also helps in increasing energy absorption. Thereafter, as crushing progressed, the core plates of egg honeycomb grid core underwent significant angular deflection (inclination) from their initial straight configuration, Fig. 3(b) (E1-III, E2-III). The individual plates of egg-honeycomb grid core structure could not maintain their vertical position as the compressive load increased due to weak constraints between neighboring grid cores. This caused increased inclination ultimately leading to failure. Note that this inclination was not perceptible for the pyramidal honeycomb grid core due to better constraining of the plates. This better constraining of individual plates of pyramidal-honeycomb grid core caused them to maintain their vertical configuration along with much better distribution of the load among the neighboring grid cores much further into the compressive regime. This resulted in superior mechanical properties of the pyramidal honeycomb grid. Each failure mode I, II, III and IV shown in Fig. 3 (b) and 4 (b), corresponds to the points indicated in the stress-strain response discussed earlier, Figs. 3 (a) and 4 (a).

The stiffness of the egg honeycomb grid $E$ can be estimated using a trapezoid plate approximation for individual grid core elements using the following relationship, 


$$
E=\frac{4 H d}{a^{2}\left(1+\frac{2 H}{b-a} \ln \frac{b H}{a H-a h+b h}\right)} E_{s}
$$

where $E_{\mathrm{s}}$ is the stiffness of the ribs, $d$ is the thickness of the plate, $b$ is the width of the top trapezoidal end, and $a$ is the width of the bottom trapezoidal end, as shown in Fig. 1(a). From our experiments, crushing of grid cores emerges as a chief mechanism of failure of the sandwich panels under out-of-plane compression. Denoting the plastic micro-buckling stress of the cell wall material in the core height direction by $\sigma_{s}$, the peak strength $\sigma_{E C}$ of the egg honeycomb grid sandwich panel can be estimated from $\sigma_{E C}=\frac{2 b d}{a^{2}} \sigma_{s}$. We use this value for the failure of trapezoidal plate in both egg and pyramidal honeycomb grid cores. The twin peaks observed in the loading curve correspond to the crushing occurring first at the top face sheet (at height $H$ ) and then at the lower face sheet (at height $h$ ) as loading progressed. Since the load bearing section for the second peak would be larger than the first ( $b$ appearing in the above expression for $\sigma_{E C}$ would be $a$, Fig. 1c), the second peak would also be corresponding greater than the first. The analytical values of the failure loads and stiffness (Eq. 2) were calculated by using $E_{\mathrm{s}}=54.504$ $\mathrm{GPa}$ and $\sigma_{\mathrm{s}}=473 \mathrm{MPa}$ which were measured experimentally using laminate with the same sequence $\left[0^{\circ} / 90^{\circ}\right]_{\mathrm{s}}$ as our trapezoidal plate in our previous paper [16]. Note that the analytical stiffness and strength of the pyramidal honeycomb grid sandwich panel would be two times of that of the egg honeycomb grid sandwich panel due to the geometry of the construction. A comparison of the stiffnesses and strengths at the first and second peak obtained from the theoretical calculations and measurements is shown in Table 1. It can be seen that the measured strengths (peak stresses) are lower than those estimated analytically. This difference can be attributed to several reasons, including voids and misaligned fibers in the cut composite plates. In addition, the slots for the cut plates can reduce the strengths of grid cores. The present 3D grid sandwich panels tended to crush rather than undergo Euler buckling which occurs in the traditional grid sandwich panels [19] at low densities.

\subsection{Energy absorption}

It is convenient to evaluate the panel's energy-absorbing ability under the out-of-plane compression. The compression curves reveal long deformation plateaus, suggesting that both the 
egg and pyramidal honeycomb grid sandwich panels may potentially be good energy-absorbing materials. The energy absorption capacity, $W$, can be calculated by integrating the compression stress-strain curve, as given by:.

$$
W=\int_{0}^{\varepsilon_{D}} \sigma(\varepsilon) d \varepsilon
$$

where $\sigma(\varepsilon)$ is the compression stress, $\varepsilon_{D}$ is the compression strain where $\varepsilon_{D}$ is the strain at the end/beginning of densification regime. In an earlier study, Fan et al [18] pointed out that the energy absorption capacities per unit mass of carbon fiber reinforced square honeycombs are much better than that of 304 stainless square honeycombs (measured at a 0.4 compressive strain). Here, we calculated the energy absorption capacities per unit mass of both the egg and pyramidal honeycomb grid sandwich panels and compared the results with those of square honeycombs [19]. As shown in Fig. 5, the 3D grid sandwich structures developed in this study have superior specific energy absorbing abilities than those of other square honeycombs with low densities (10-100 $\mathrm{Kg} / \mathrm{m}^{3}$ ). This can be explained by noting, that for the traditional square honeycombs, Euler buckling failure can occur more readily at low core densities. Since the effective of energy absorption due to Euler buckling is much lower than the energy absorption due to crushing failure occurring in our three dimensional honeycomb grids, lesser energy is absorbed in the former case. Interestingly, at the same relative $\operatorname{den} \operatorname{sity}(\bar{\rho}=6.0 \%)$, the specific energy absorption of the pyramidal honeycomb grid sandwich panel is slightly higher than that of the egg honeycomb grid sandwich panel due to the better stability of the pyramidal honeycomb grid core.

\section{Conclusions}

In this letter, carbon fiber reinforced composite sandwich panels made with egg and pyramidal honeycomb grid cores were designed and manufactured by the interlocking method. The out-of-plane compression tests and analytical calculations were carried out to study their mechanical response. The failure modes of the specimens were photographed and identified. It was found that crushing is the main failure mode of 3D grid core sandwich panels under out-of-plane compression even at low core densities. The existence of the relatively long plateau stage due to the progressive failure of core plates in the compression curves endows them with higher specific energy absorbing abilities compared to other square honeycombs with low densities $\left(10^{1}-10^{2} \mathrm{Kg} / \mathrm{m}^{3}\right)$. The present study provides a novel method to fabricate carbon fiber 
reinforced composite grids cores with interconnected void spaces, which can be used to design and develop lightweight structures with superior energy absorption ability and multifunctional capabilities.

\section{Acknowledgements}

The present work was supported in part by the National Science Foundation of China under grant Nos. 11302060 and 11572100, Natural Scientific Research Innovation Foundation in Harbin Institute of Technology (HIT.NSRIF.2014025) and in part by NSF-CAREER Grant No. CMMI-1149750 (AV and RGh),

\section{References}

[1] Pan SD, Wu LZ, Sun YG, et al. Fracture test for double cantilever beam of honeycomb sandwich panels. Mater Letters 2008; 62: 523-526.

[2] Vaziri A, Xue Z, Hutchinson JW. Metal sandwich plates with polymeric foam-filled cores. J. Mech. Mater. Struct 2006, 1(1): 95-128.

[3] Qin QH, Wang TJ. Low-velocity impact response of fully clamped metal foam core sandwich beam incorporating local denting effect. Compo Struct 2013; 96: 346-356.

[4] Xiong J, Wu LZ, Ma L, et al. Fabrication and crushing behavior of low density carbon fiber composite pyramidal truss structures. Compo. Struct 2010; 92: 2695-2702.

[5] Joo JH, Kang KJ, Kim T, et al. Forced convective heat transfer in all metallic wire-woven bulk Kagome sandwich panels. Int J Heat Mass Trans 2011; 54: 5658-5662.

[6] Liu JY, Xiang LL, Kan T. The effect of temperature on the bending properties and failure mechanism of composite truss core sandwich structures. Compo. Part A 2015; 79: 146-154.

[7] Vaidya UK, Hosur MV, Earl D, et al. Impact response of integrated hollow core sandwich composite panels. Compos. Part A 2000; 31: 761-772.

[8] Gao L, Sun YG. Fluid flow and heat transfer characteristics of composite lattice core sandwich structures. J Thermophys Heat Tr 2014; 28(2): 258-269.

[9] Ullah I, Brandt M, Feiha S. Failure and energy absorption characteristics of advanced 3D truss core structures. Mater. Des. 2016; 92: 937-948.

[10] Chen M, Zhu X, Lei H, et al. Optimal design of broadband radar absorbing sandwich structure with circuit analog absorber core. Int J Appl Mech 2015; 7(02): 1550020.

[11] Ebrahimi H, Vaziri A. Metallic sandwich panels subjected to multiple intense shocks. Int J Solids Struct 2013; 50: 1164-1176.

[12] Vaziri A, Hutchinson JW. Metallic sandwich plates subject to intense air shocks. Int J Solids Struct 2007; 44: 2021-2035. 
[13] Xin FX, Lu TJ. Analytical modeling of fluid loaded orthogonally rib-stiffened sandwich structures: Sound transmission. J Mech Physics Solids 2010; 58: 1374-1396.

[14] Xin FX, Lu TJ. Sound radiation of orthogonally rib-stiffened sandwich structures with cavity absorption. Compos Sci Tech 2010; 70: 2198-2206.

[15] Kim T, Zhao CY, Lu TJ, et al. Convective heat dissipation with lattice frame materials. Mech Mater 2004; 36: 767-780.

[16] Xiong J, Zhang M, Stocchi A, et al. Mechanical behaviors of carbon fiber composite sandwich columns with three dimensional honeycomb cores under in-plane compression. Compos. Part B: Eng 2014; 60: 350-358.

[17] Xiong J, Ma L, Stocchi A, et al. Bending response of carbon fiber composite sandwich beams with three dimensional honeycomb cores. Compo. Struct 2014; 108: 234-242.

[18] Fan HL, Meng FH, Yang W. Sandwich panels with Kagome lattice cores reinforced by carbon fibers. Compos Struct 2007; 81: 533-539.

[19] Russell BP, Deshpande VS, Wadley HNG. Quasistatic deformation and failure modes of composite square honeycomb. J Mech Mater Struct 2008; 3:1315-1340. 


\section{Figures}

Fig.1. Fabrication of 3D sandwich cores using the interlock method. (a) Composite sheet shapes after cutting and prior to assembly, (b) Assembled composite sheets for each core construction, (c) Unit-cells of each core construction, where geometrical parameters that define core size are shown, (d) Photographs of fabricated grid cores (egg honeycomb grid: $\bar{\rho}=3.0 \%$; pyramidal honeycomb grid: $\bar{\rho}=6.0 \%$.)

Fig. 2 Sandwich panel made with carbon fiber reinforced composite pyramidal honeycomb grid core $(\bar{\rho}=12.0 \%)$.

Figure 3 (a) the compressive stress-strain responses of sandwich panels with egg honeycomb grid cores, (b) photographs of compressive failure modes of egg honeycomb grid cores with $\bar{\rho}=3.0 \%$ (top) and $\bar{\rho}=6.0 \%$ (bottom).

Figure 4 (a) the compressive stress-strain responses of sandwich panels with pyramidal honeycomb grid cores, (b) photographs of compressive failure modes of pyramidal honeycomb grids cores with $\bar{\rho}=6.0 \%$ (top) and $\bar{\rho}=12.0 \%$ (bottom).

Fig.5. A comparison between the energy absorption capacities per unit mass (up to a compressive strain $\varepsilon=0.4$ ) of competing metallic and composite grids sandwich panels, as reported in [19].

\section{Table}

Table 1 The out-of-plane compression properties of carbon fiber reinforced composite 3D grids sandwich panels. Experimental results associated with both first and second peaks in loading, as well as analytical estimates are shown. 
(a) Plate I

Trapezium plate
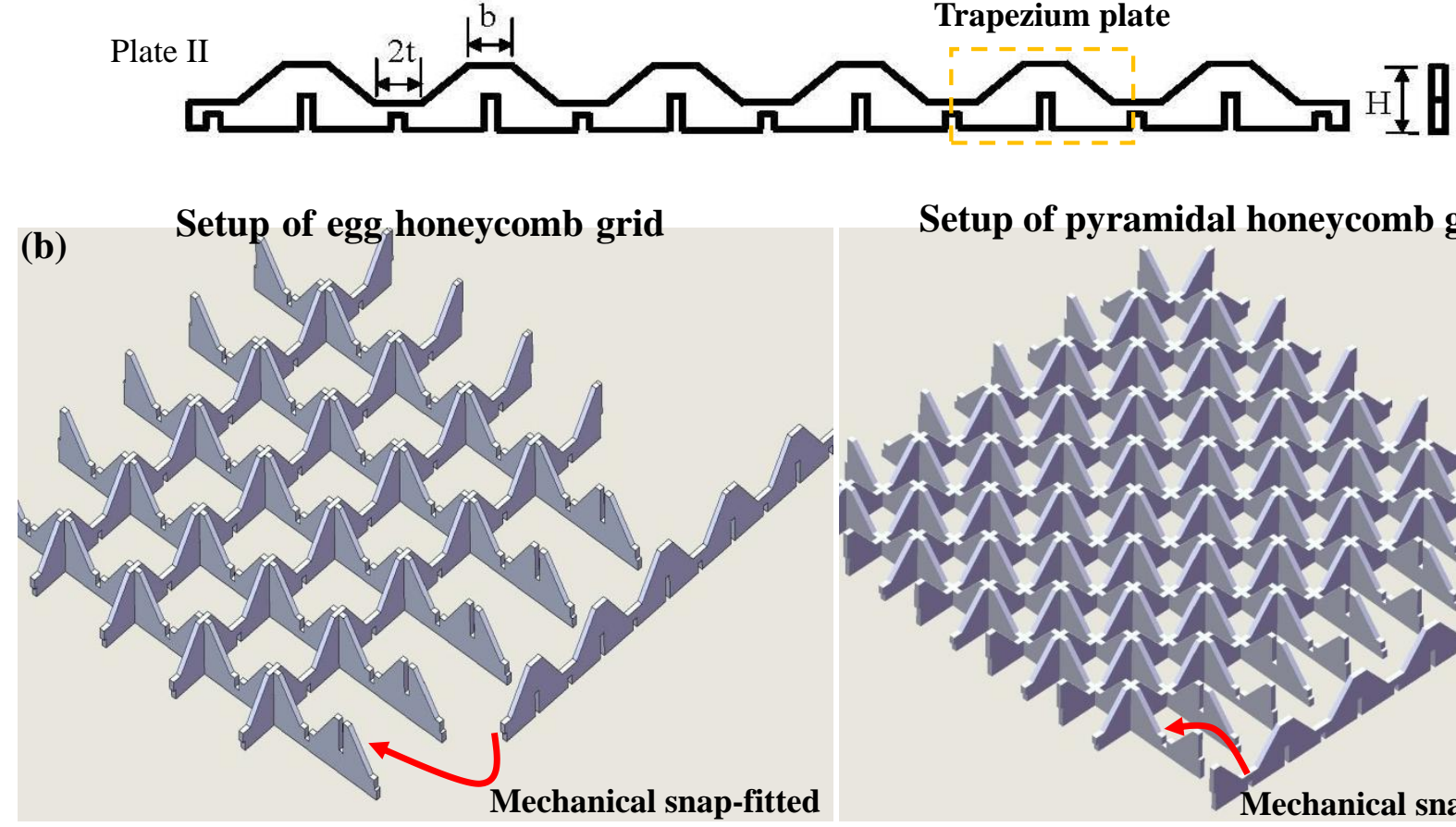

Setup of pyramidal honeycomb grid core

(c)
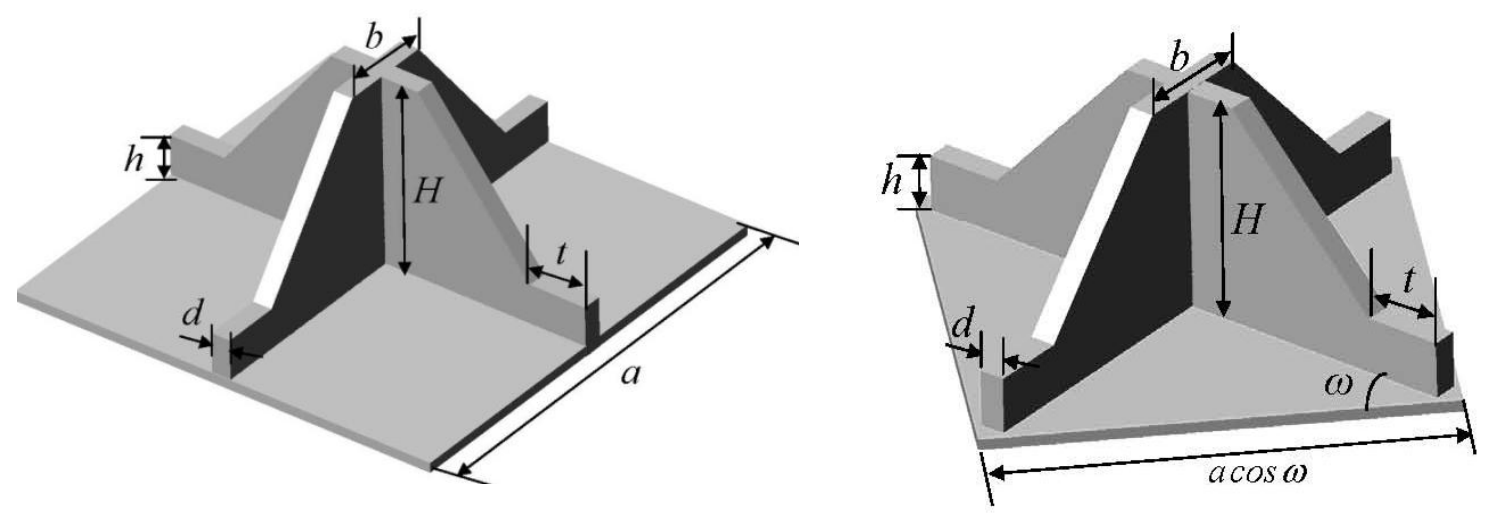

(d) Egg honeycomb grid core fabricated

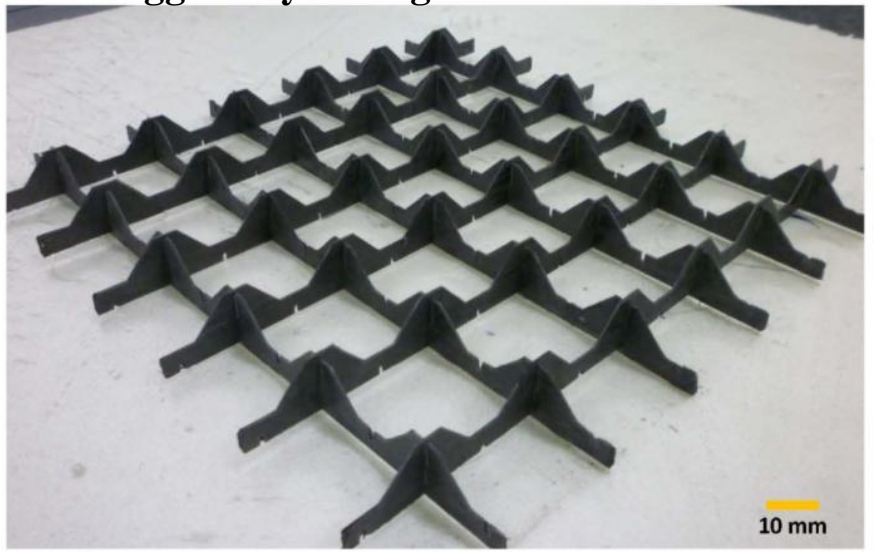

Pyramidal honeycomb grid core fabricated

Figure 1

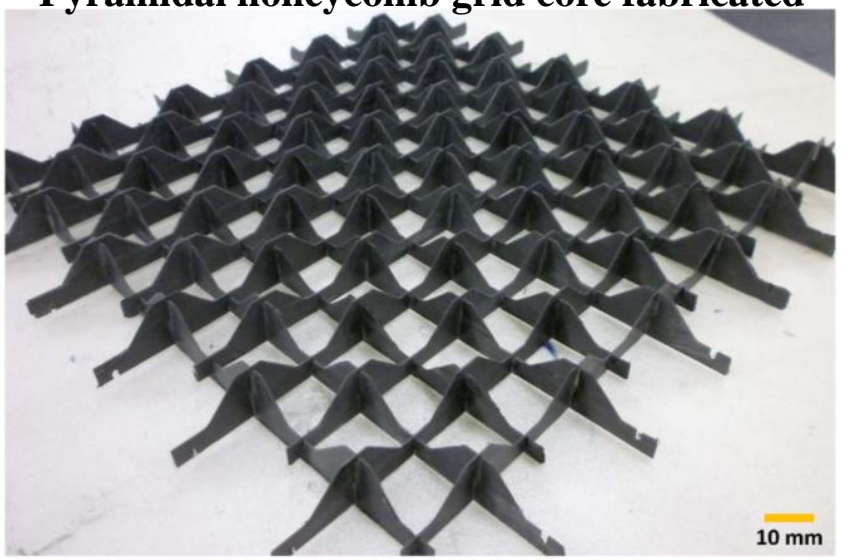




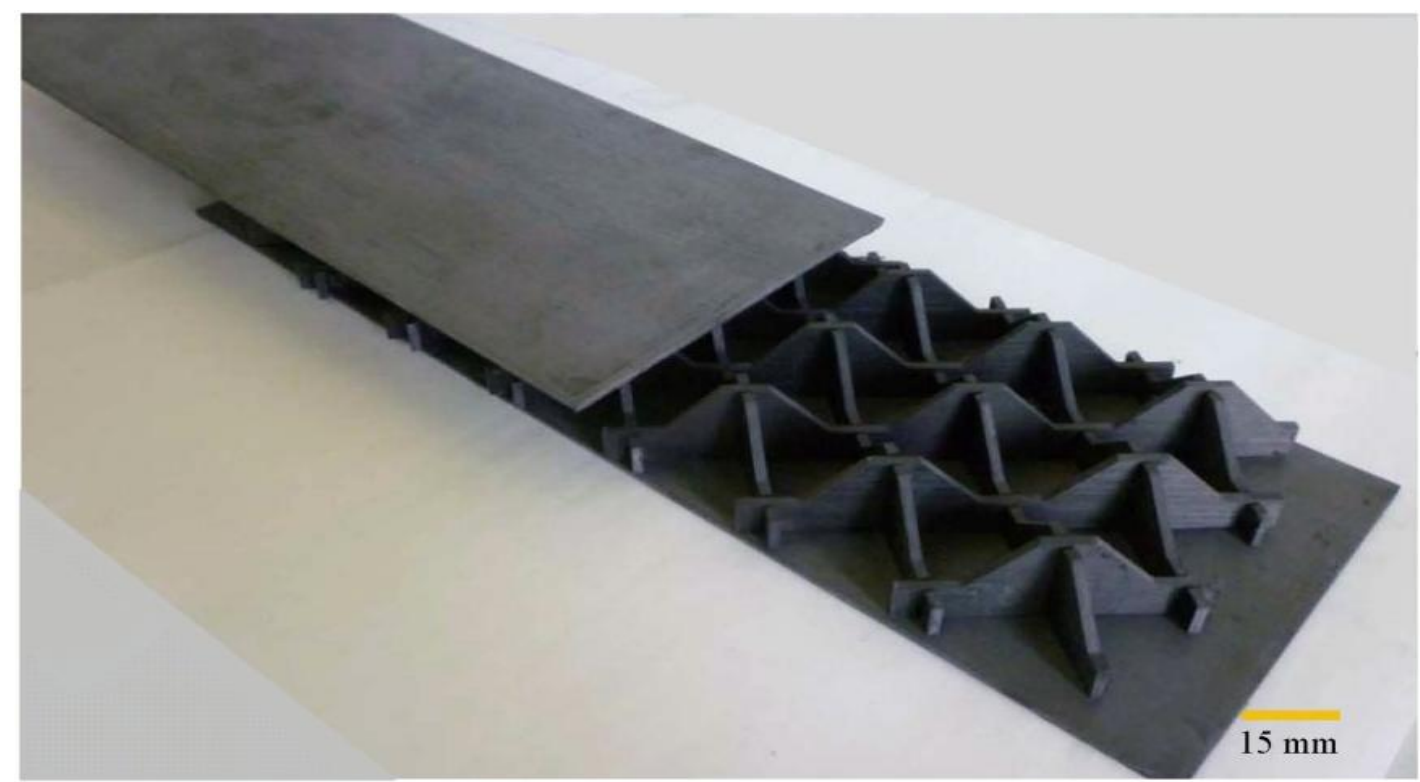

Figure 2 


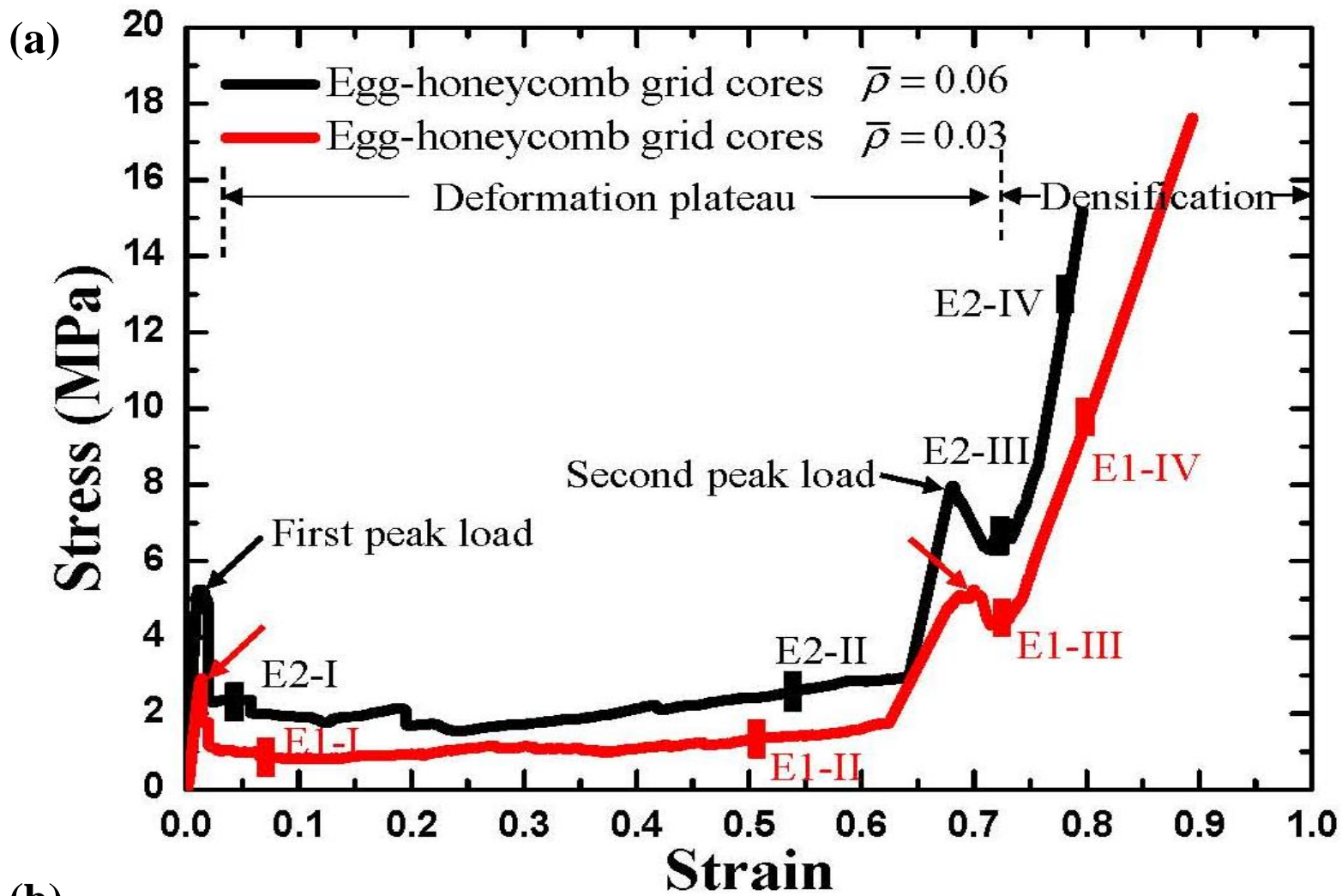

(b)
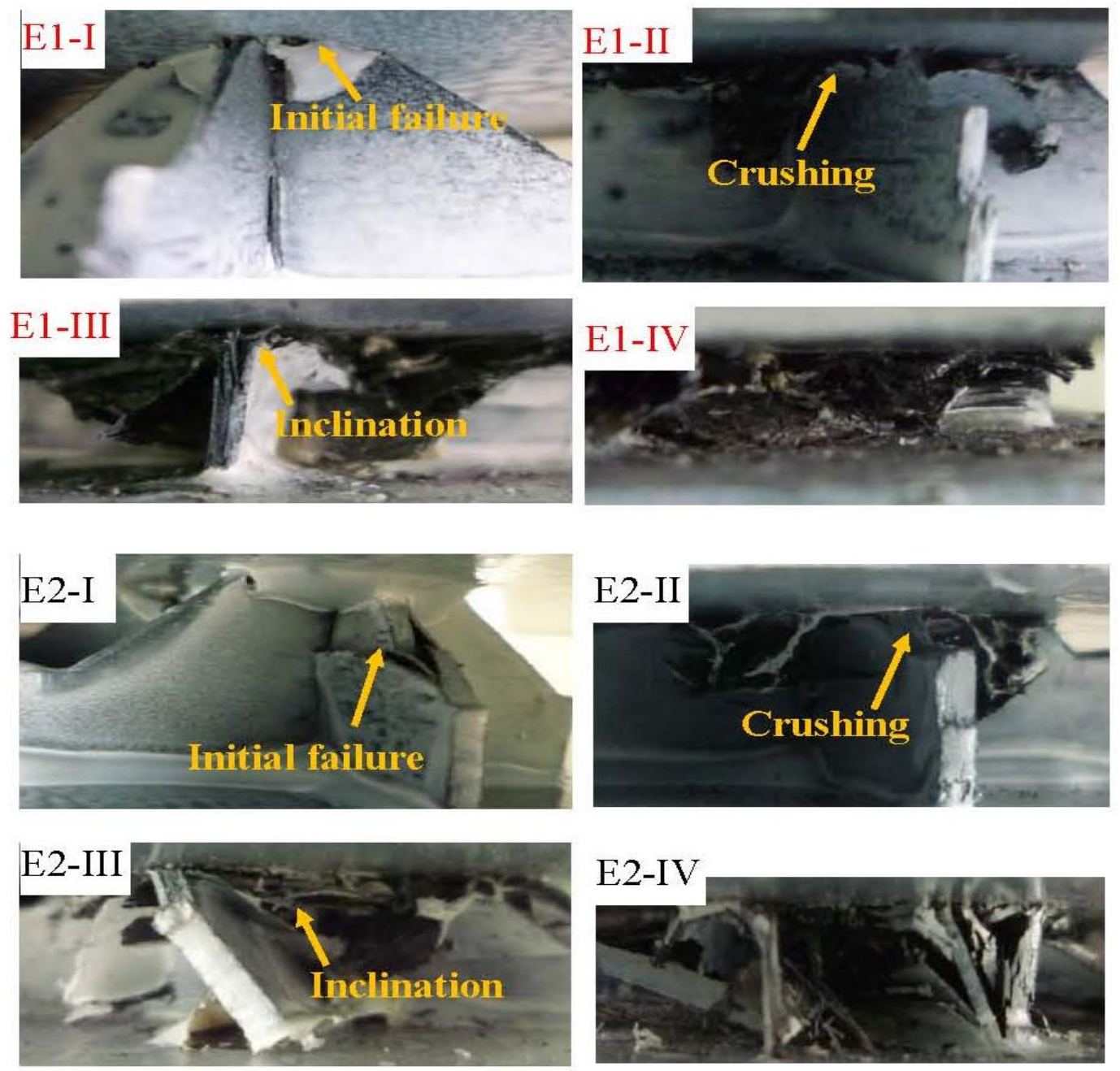

Figure 3 
(a)

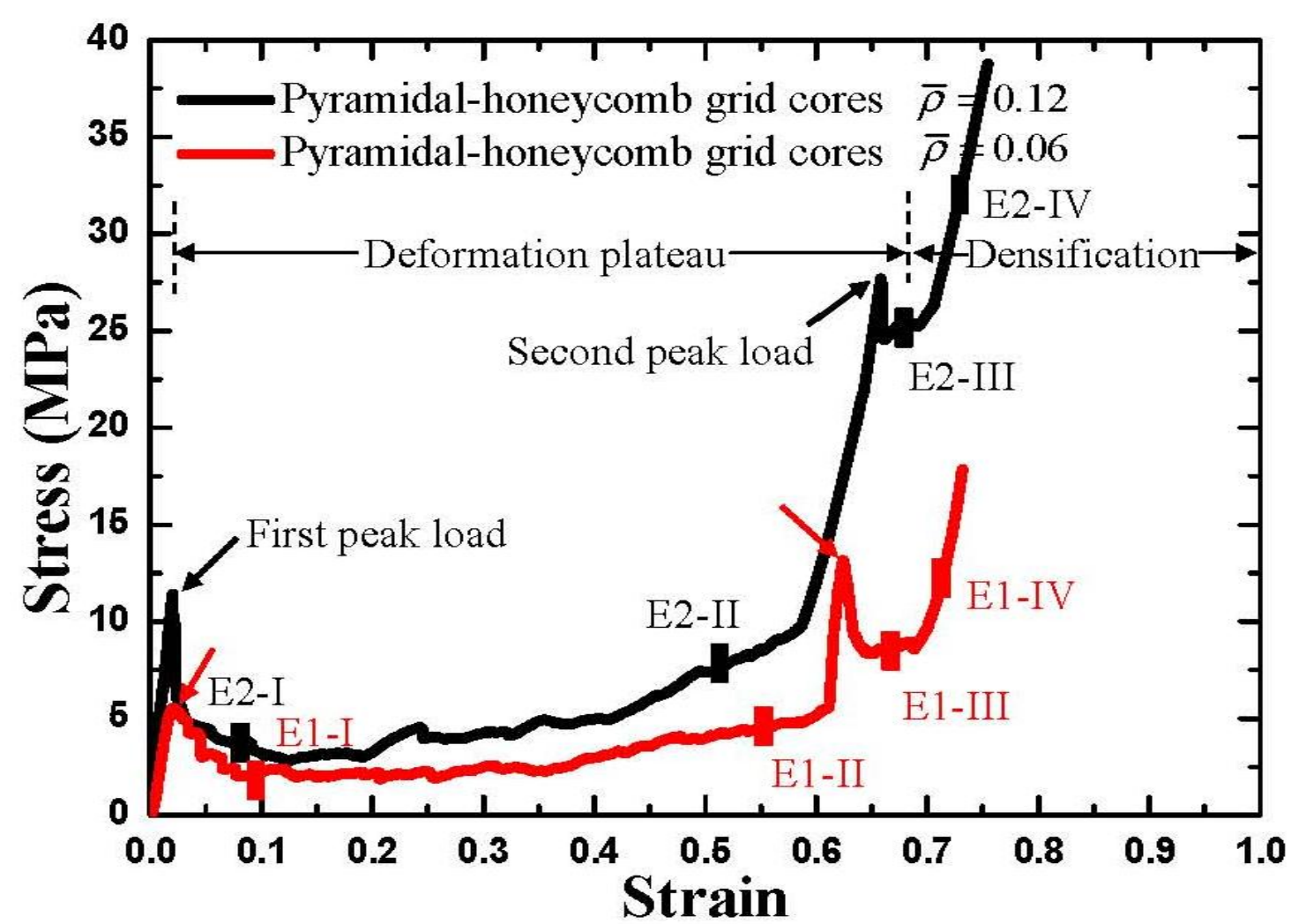

(b)
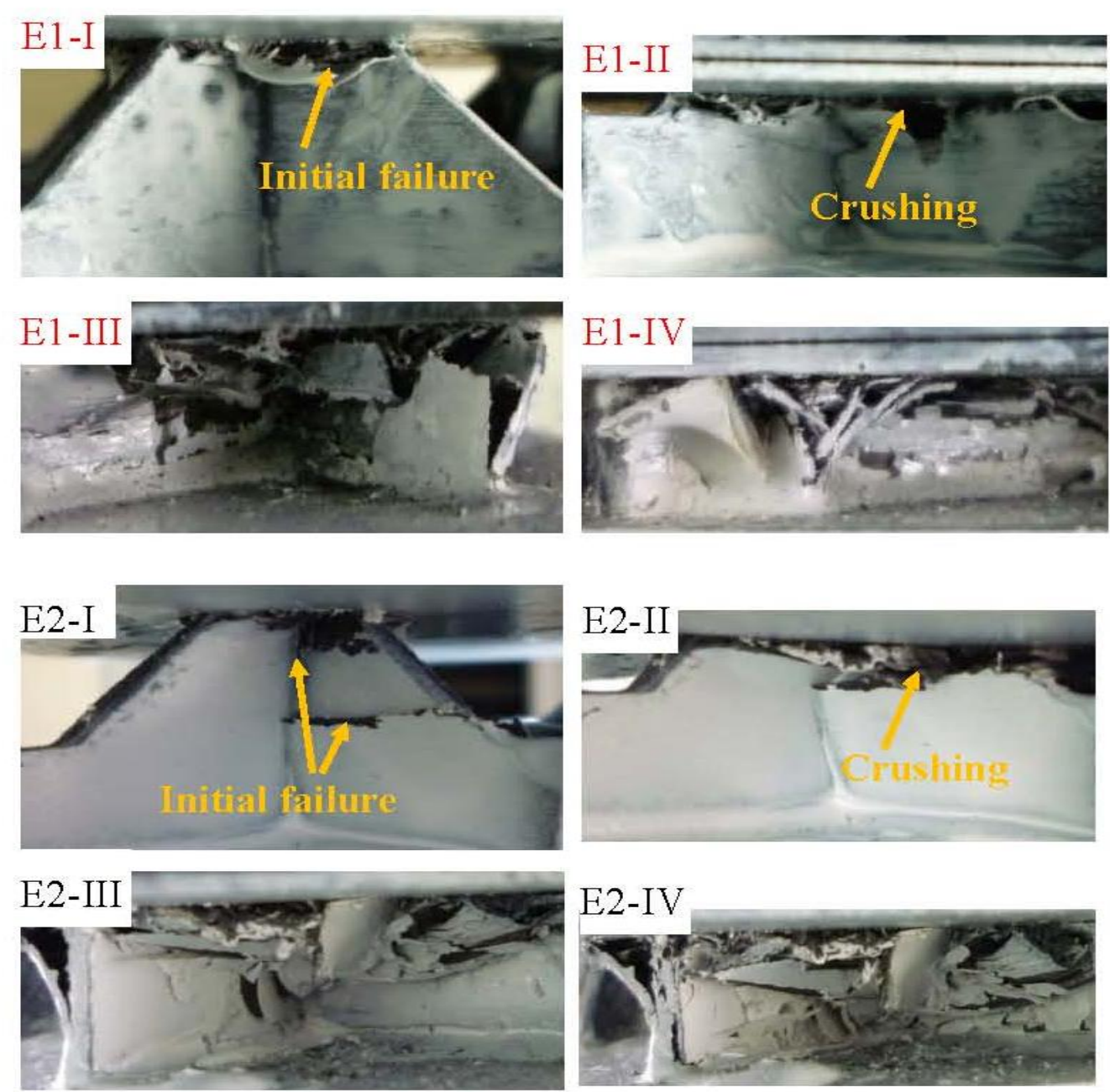

Figure 4 


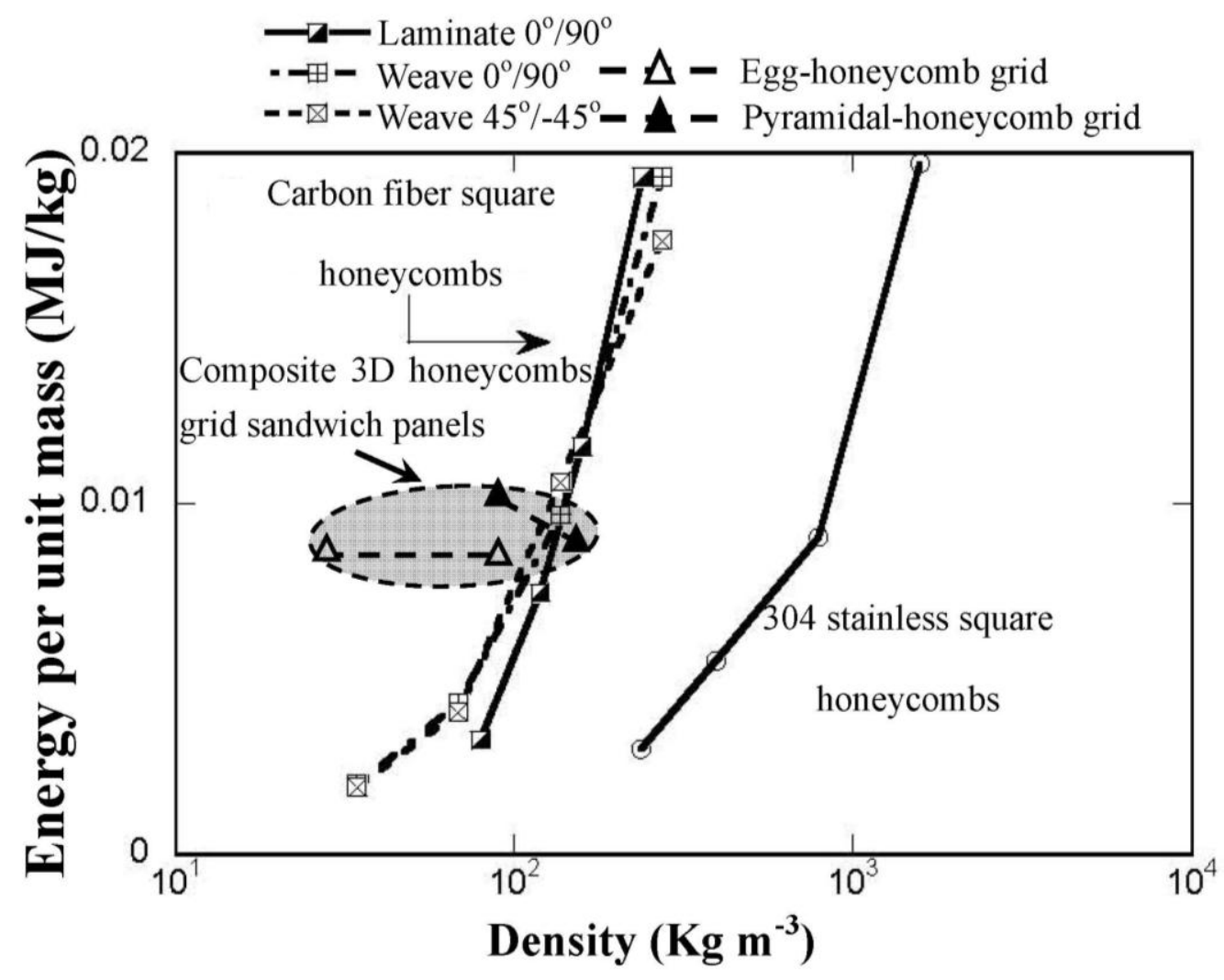

Figure 5

Table 1 The out-of-plane compression properties of carbon fiber reinforced composite 3D grids sandwich panels. Experimental results associated with both first and second peaks in loading, as well as analytical estimates are shown.

\begin{tabular}{|c|c|c|c|c|c|c|}
\hline Specimens & $\begin{array}{c}d \\
(\mathrm{~mm})\end{array}$ & $\begin{array}{l}\text { Relative } \\
\text { density }\end{array}$ & $\begin{array}{l}\text { Density } \\
\left(\mathrm{Kg} / \mathrm{m}^{3}\right)\end{array}$ & $\begin{array}{l}\text { First pea } \\
\text { Second }\end{array}$ & $\begin{array}{l}\text { Obs. Stiffness/ Strength } \\
\text { ak-Obs. Strength } \\
\text { (MPa) }\end{array}$ & $\begin{array}{l}\text { Analyt. } \\
\text { Stiffness/ } \\
\text { Strength(MPa) }\end{array}$ \\
\hline \multirow{4}{*}{$\begin{array}{c}\text { Egg } \\
\text { honeycomb } \\
\text { grid panel }\end{array}$} & \multirow[t]{2}{*}{1} & \multirow[t]{2}{*}{0.03} & \multirow[t]{2}{*}{46.5} & First & $485( \pm 30) / 2.92( \pm 0.30)$ & $908.66 / 4.34$ \\
\hline & & & & Second & $5.26( \pm 0.61)$ & 20.79 \\
\hline & \multirow[t]{2}{*}{2} & \multirow[t]{2}{*}{0.06} & \multirow[t]{2}{*}{93} & First & $995( \pm 26) / 5.25( \pm 0.52)$ & $1817.32 / 8.69$ \\
\hline & & & & Second & $7.95( \pm 0.55)$ & 41.57 \\
\hline \multirow{4}{*}{$\begin{array}{l}\text { Pyramidal } \\
\text { honeycomb } \\
\text { grid panel }\end{array}$} & \multirow{2}{*}{1} & \multirow{2}{*}{0.06} & \multirow{2}{*}{93} & First & $975( \pm 22) / 5.54( \pm 0.43)$ & $1817.32 / 8.69$ \\
\hline & & & & Second & $13.16( \pm 0.85)$ & 41.58 \\
\hline & \multirow{2}{*}{2} & \multirow{2}{*}{0.12} & \multirow{2}{*}{186} & First & $1918( \pm 49) / 11.41( \pm 0.63)$ & $3634.65 / 17.37$ \\
\hline & & & & Second & $27.68( \pm 1.04)$ & 83.14 \\
\hline
\end{tabular}

